\title{
Evaluation of the dosimetric impact of loss and displacement of seeds in prostate low-dose-rate brachytherapy
}

\author{
Yinkun Wang, MScl, Nicola J. Nasser, MD, PhD², Jette Borg, PhD!', Elantholi P. Saibishkumar, MBBS, MD, FRCPC 3,4 \\ 'Department of Radiation Physics, Princess Margaret Cancer Centre, University Health Network, Toronto, Ontario, Canada, ${ }^{2}$ Department of \\ Radiation Oncology, Memorial Sloan- Kettering Cancer Centre, New York, New York, USA, ${ }^{3}$ Department of Radiation Oncology, Princess \\ Margaret Cancer Centre, University Health Network, Toronto, Ontario, Canada, ${ }^{4}$ Department of Radiation Oncology. University of Toronto, \\ Toronto, Ontario, Canada
}

\begin{abstract}
Purpose: To analyze the seed loss and displacement and their dosimetric impact in prostate low-dose-rate (LDR) brachytherapy while utilizing the combination of loose and stranded seeds.

Material and methods: Two hundred and seventeen prostate cancer patients have been treated with LDR brachytherapy. Loose seeds were implanted in the prostate center and stranded seeds in the periphery of the gland. Patients were imaged with transrectal ultrasound before implant and with computerized tomography/magnetic resonance imaging (CT/MR) one month after implant. The seed loss and displacement had been analyzed. Their impact on prostate dosimetry had been examined. The seed distribution beyond the prostate inferior boundary had been studied.

Results: The mean number of seeds per patient that were lost to lung, pelvis/abdomen, urine, or unknown destinations was $0.21,0.13,0.03$, and 0.29 , respectively. Overall, $40.1 \%$ of patients had seed loss. Seed migration to lung and pelvis/abdomen occurred in $15.5 \%$ and $10.5 \%$ of the patients, respectively. Documented seed loss to urine was found in $3 \%$ of the patients while $20 \%$ of patients had seed loss to unknown destinations. Prostate length difference between pre-plan and post-implant images was within $6 \mathrm{~mm}$ in more than $98 \%$ of cases. The difference in number of seeds inferior to prostate between pre-plan and post-implant dosimetry was within 7 seeds for $93 \%$ of patients. At time of implant, $98 \%$ of seeds, inferior to prostate, were within $5 \mathrm{~mm}$ and $100 \%$ within $15 \mathrm{~mm}$, and in one month post-implant $83 \%$ within $9 \mathrm{~mm}$ and $96.3 \%$ within $15 \mathrm{~mm}$. Prostate post-implant $\mathrm{V}_{100}, \mathrm{D}_{90}$, and rectal wall $\mathrm{RV}_{100}$ for patients without seed loss were $94.6 \%, 113.9 \%$, and $0.98 \mathrm{~cm}^{3}$, respectively, as compared to $95.0 \%, 114.8 \%$, and $0.95 \mathrm{~cm}^{3}$ for the group with seed loss.
\end{abstract}

Conclusions: Seed loss and displacement have been observed to be frequent. No correlation between seed loss and displacement and post-plan dosimetry has been reported.

Key words: brachytherapy, dosimetry, LDR, prostate cancer, seeds.

\section{Purpose}

Low-dose-rate (LDR) brachytherapy utilizing permanent implant of radioactive seeds is a highly effective treatment option for low and intermediate risk prostate cancer patients [1-3]. In LDR brachytherapy treatment for prostate cancer patients, seed loss and seed displacement are well-recognized [4-12]. Due to inaccuracies in seed placement, blood flow, muscular forces, and edema post-implant, the implanted seeds may experience redistribution and displacement from their intended positions. Although seeds may migrate in any direction, they tend to move more easily along the needle track in the superior- inferior direction [13]. Some of the implanted seeds could even migrate to lung, abdomen, pelvis, or get excreted in urine. In some cases, even after extensive investigation and imaging, the destination of part of the seeds could not be confirmed.

In LDR prostate brachytherapy loose seeds and/or stranded seeds are used. Seed loss and migration after implant and their dosimetric effect have been studied by a number of groups. Taussky et al. studied 495 patients treated with I-125 loose seeds using an automated seed delivery system and found that the percentage of patients experiencing seed loss gradually decreased from $38 \%$ for

Address for correspondence: Saibishkumar Elantholi Parameswaran, M.B.B.S, MD, FRCPC, Department Received: 10.01 .2015 of Radiation Oncology, Princess Margaret Cancer Centre, University Health Network, 610 University Ave., Accepted: 21.05 .2015 Toronto, Ontario, Canada M5G 2M9, phone: +1 416-946-2919, fax: +1 416-946-6561, Published: 25.06.2015

e-mail: saibish.elantholi@rmp.uhn.on.ca 
the first 100 patients to $9 \%$ for the last 100 patients as they gained more experience [4]. Gao et al. simulated the effects of seed migration on post-implant dosimetry based on 14 patients treated with loose Pd-103 seeds [5]. They concluded that on average prostate $\mathrm{D}_{90}$ will decrease $1 \%$ in $2 \mathrm{~mm}$ mean migration and $6 \%$ in $6 \mathrm{~mm}$ mean migration. Prostate $V_{100}$ was less sensitive to migration than $D_{90}$. They also found that the range of the dose change depends on the distance of seed migration. Knaup et al. reported their studies on five patients' plans, each of them planned with I-125, Pd-103, and Cs-131 loose seeds [6]. Their simulation was done by removing 1, 2, or 3 seeds closest to urethra or the exterior prostatic capsule. They found a reduction of $2 \%, 5 \%$, and $7 \%$ in $D_{90}$ for loss of 1 , 2 , or 3 seeds through the urethra, respectively. They also found that the dosimetric effect of seed loss near the exterior of the prostate through the prostatic veins was less severe than that of seed loss through the urethra. Pinkawa et al. studied 61 patients treated with stranded seeds [7]. Inferior displacements were found between 2.2 and $5.3 \mathrm{~mm}$ for four groups of seed locations. The comparison of seed loss and migration between loose seeds and stranded seeds was also investigated by several authors. Fuller et al. found that stranded seeds were associated with decreased seed migration and higher prostate and urethra dosimetry values when compared to loose seeds [8]. Saibishkumar et al. reported that the overall seed loss was greater in the stranded seed cohort than that in the loose seed cohort (1.1\% of total seeds vs. $0.6 \%$ ) [9]. Kaplan et al. found that the average radial deviation of the loose seeds from the planned position was $3.1 \mathrm{~mm}$, while that of the suture embedded seeds was $3.7 \mathrm{~mm}$, and there was no improvement in the final dosimetry when suture embedded seeds were used [10]. Ishiyama et al. compared pre-plan technique, intraoperative pre-plan technique, and interactive plan technique and found no significant difference in biochemical control among the three groups but the interactive plan showed a significant reduction of the seed migration rate compared to the two other groups [12].

In the above investigations, particular emphasis was placed on either loose seeds or stranded seeds. Recently, Langley et al. reported a new one-stage prostate brachytherapy technique using a combination of stranded and loose seeds [14]. Their data showed significantly improved dosimetry $\left(D_{90}\right.$ and $\left.V_{100}\right)$ for the techniques using both stranded and loose seeds when compared to stranded seeds only. In this study, we analyzed the seed loss and displacement in prostate cancer patients treated with LDR brachytherapy with loose seeds implanted in the prostate center and stranded seeds in the periphery of the gland. The dosimetric effect caused by the seed loss and displacement was also examined.

\section{Material and methods}

\section{Patient population}

In this retrospective study, data from 217 consecutive low- and intermediate-risk prostate patients treated between March 2009 and December 2012 with LDR brachytherapy at Princess Margaret Cancer Centre were analyzed. Eligibility criteria for the study were patients aged eighteen years or older with histologically confirmed prostate cancer, and no evidence of metastases. All patients studied had pretreatment planning utilizing transrectal ultrasound (TRUS), implant procedure with I-125 seeds, prescribed dose of $145 \mathrm{~Gy}$, and post-implant dosimetry at one month based on magnetic resonance imaging (MRI) and computerized tomography (CT) pelvic scans.

\section{Treatment planning}

All patients were imaged with BK ProFocus trans-rectal ultrasound (TRUS) machine (BK Medical ApS, Herlev, Denmark) for prostate brachytherapy treatment planning and volume study 2-4 weeks prior to implant. The scanning was performed at $9 \mathrm{MHz}$ transducer frequency. Transverse images were recorded at $5 \mathrm{~mm}$ slice spacing and then downloaded to the treatment planning system. Treatment planning was conducted on VariSeed (Varian Brachytherapy, Charlottesville, VA, USA) using modified peripheral loading technique. The prescription was 145 Gy minimum peripheral dose (mPD) to prostate [15]. The clinical target volume was defined as the prostate with an anterior and lateral margin of $3 \mathrm{~mm}$, and a $5 \mathrm{~mm}$ margin in the cranial and caudal directions respecting anatomic boundaries like bladder wall and rectal wall. There was no posterior margin extended towards the rectum. For intermediate risk prostate cancer patients, lateral margins were $5 \mathrm{~mm}$. Prostate contours were drawn and plans utilizing seeds with a mean air kerma strength of $0.435 \pm 0.036 \mathrm{U}$ were placed on images with $5 \mathrm{~mm}$ spacing. The measured prostate lengths were not in increments of $5 \mathrm{~mm}$, so depending on the measured length of prostate, seeds were planned 2.5 to $7.5 \mathrm{~mm}$ below prostate.

The dosimetry goals for pre-plans were to achieve prostate $V_{100}$ (the percentage of the prostate volume receiving $100 \%$ of the prescribed dose or more) greater than $99 \%, \mathrm{D}_{90}$ (the percentage of the prescribed dose received by $90 \%$ volume of the prostate) between $120 \%$ and $125 \%$ of the prescription dose, and $\mathrm{V}_{150}$ (the percentage of the prostate volume receiving $150 \%$ of the prescribed dose or more) between $55 \%$ and $62 \%$. For organs at risk, the urethral dose was to keep $\mathrm{UD}_{5}$ (the dose that is received by $5 \%$ volume of the prostatic urethra) under $150 \%$ and $\mathrm{UD}_{30}$ (the dose that is received by $30 \%$ volume of the prostatic urethra) limited to $125 \%$ of the prescription dose $[16,17]$. $\mathrm{RV}_{100}$ (the absolute volume of the rectal wall to receive $100 \%$ of the prescription dose or more) was planned to be less than $1.0 \mathrm{~cm}^{3}$.

\section{Implant procedure}

Permanent seed implantation was performed under general anesthesia. The patient was positioned in lithotomy as close to the exact position as possible as at the time of the mapping session. Under TRUS guidance, trans-perineal insertion of seeds utilizing template and needles was performed according to the pre-treatment plan. For each patient, I-125 loose seeds (Oncura 6711, Oncura-GE Healthcare, Arlington Heights, IL, USA) were implanted in the prostate center, while I-125 stranded seeds (Oncura 
6711 RAPID strands, Oncura-GE Healthcare, Arlington Heights, IL, USA) were used on the prostate periphery. During the procedure, serial X-ray imaging of prostate was obtained after each row of seeds was implanted to assess the quality of seed insertion. An intra-operative decision to add seeds on top of the pre-treatment plan was done after review of ultrasound and X-ray images and involved mostly the addition of one or two seeds.

\section{Post-implant evaluation}

Anterior-posterior and lateral chest $\mathrm{X}$-rays were taken one month after implant. Post-implant dosimetry, using CT-MRI fusion, was performed 30 days after the implant. Axial CT images were taken for the entire pelvis in the supine position with Aquilion ONE Toshiba CT scanner (Toshiba America Medical Systems, Inc., Tustin, CA, USA) after insertion of a urethral Foley catheter. Slices were obtained at $3 \mathrm{~mm}$ intervals without an interslice gap. Axial MR scans for part of the pelvis but larger than the prostate were obtained using 3T IMRIS/Siemens MRI scanner (IMRIS, Winnipeg, MB, Canada), and were obtained immediately before or after the CT. The slice thickness was $3 \mathrm{~mm}$ with no interslice gap. Computed tomography spatial resolution was $0.4-0.6 \mathrm{~mm}$, and MR spatial resolution was $0.7-1.0 \mathrm{~mm}$. Computerized tomography/magnetic resonance imaging fusion was performed manually by the brachytherapy dosimetrist, relying on the brachytherapy seeds as fiducial markers. Seed location was determined with VariSeed ${ }^{\mathrm{TM}}$ on the CT images and number of seeds was verified against seeds counted on pelvic X-ray images. Patients were expected to strain their urine for 3 days following the implant procedure. All relevant soft tissue structures were contoured on the MR images, except the urethra (Foley), which was contoured on CT images. The accuracy of the CT-MRI fusion and the smoothness of the prostate contouring were verified on the axial, coronal, and sagittal planes for each patient. Review, modification, and approval of all pre- and post-implant contouring, and the implant procedures were carried out by the same experienced physician (EPS). The seed distributions beyond the prostate inferior boundary in the $\mathrm{CT}$, and ultrasound images were analyzed and compared. Prostate lengths from both pre- and post-implant and the difference between them were studied to separate the effect of change in contour length from seed displacement. Pre- and postimplant dosimetry parameters including prostate $\mathrm{V}_{100}$ and $\mathrm{D}_{90}$ were examined for plan evaluation and assessment of impact caused by seed loss and displacement. A postplan with prostate $V_{100}$ greater than $85 \%$ and prostate $D_{90}$ greater than $90 \%$ was considered optimal [18]. The toler- ance for post-implant $R V_{100}$ was set at $1.3 \mathrm{~cm}^{3}$ to count for the changes from pre-implant in rectal proximity with resolution of periprostatic edema [16].

\section{Results}

\section{Seed loss}

The mean number of implanted seeds per patient was 110.2 (range: $75-155$, standard deviation or SD: 16.4). The mean number of seeds per patient lost to lung, pelvis/abdomen, urine, or unknown destination ( \pm SD) was $0.21 \pm 0.55,0.13 \pm 0.41,0.03 \pm 0.17$, and $0.29 \pm 0.66$, respectively. The number of seeds lost per patient, combining all destinations was $0.67 \pm 1.06$. Overall, $40.1 \%$ of patients experienced some kind of seed loss. Seed lost to lung occurred in $15.5 \%$ of the patients. In $10.5 \%$ of the patients, there was seed lost to the pelvis or the abdomen. Documented seed loss in urine occurred in 3\% of the patients, while $20 \%$ of patients have seed lost to unknown destination. Table 1 summarized the seed loss results. Examples of seed loss to lung and pelvis are shown in Fig. 1.

Overall, the trend of seeds lost against the seeds implanted was toward the decrease. This is evident as shown in Fig. 2, where the y-axis is the number of seeds lost, and the $x$-axis is the number of seeds implanted, both accumulated over time. For the first 20 patients, the slope of the linear fit was 0.0145 and was much steeper than that for the next 30 implants (0.0074). The slope of the linear fit was further decreased to 0.0053 for the remaining implants. The mean number of seeds lost per patient $( \pm$ SD) was $1.35 \pm 1.69,0.77 \pm 0.94$, and $0.57 \pm 0.96$ for the first 20 , the next 30 , and the remaining patients, respectively.

The relationship between seed loss and the prostate volume post implant is shown in Fig. 3. The prostate volume ranged from 19.9 to $87.5 \mathrm{~cm}^{3}$ with a mean $( \pm S D)$ of $42.5 \pm 12.0 \mathrm{~cm}^{3}$, and was grouped with a bin size of $10 \mathrm{~cm}^{3}$. The number of seed loss per patient was $0.00,0.17,0.55$, $0.82,1.00,0.80,1.00$, and 1.50 for the groups with a volume from the first bin (between 10 to $20 \mathrm{~cm}^{3}$ ), to the last bin (between 80 to $90 \mathrm{~cm}^{3}$ ), respectively. The seed loss rate (the ratio of the number of seeds lost to the number of seeds implanted) was $0.0 \%, 0.2 \%, 0.5 \%, 0.7 \%, 0.8 \%, 0.6 \%, 0.7 \%$, and $1.0 \%$ for the corresponding groups, respectively. The mean seed loss rate of all prostate volume groups was $0.6 \pm 0.2 \%$.

\section{Seeds inferior to prostate and seed displacement}

Prostate length prior to the seed implantation procedure, measured by TRUS, was compared to prostate length one month after the procedure measured on CT/ MR images. This analysis was performed to account for

Table 1. The mean number of seeds lost to lung, pelvis and abdomen, urine, and unknown destination per patient, and the percentage of patients experiencing seed loss to each of the above destinations

\begin{tabular}{lccccc} 
& \multicolumn{5}{c}{ Destination } \\
\cline { 2 - 7 } Seed loss & Lung & Pelvis/Abdomen & Urine & Unknown & Overall \\
\hline Mean number per patient & 0.21 & 0.13 & 0.03 & 0.29 & 0.67 \\
\hline Standard deviation & 0.55 & 0.41 & 0.17 & 0.66 & 1.06 \\
\hline Percentage of patients (\%) & 15.5 & 10.5 & 3.0 & 20.0 & 40.1
\end{tabular}



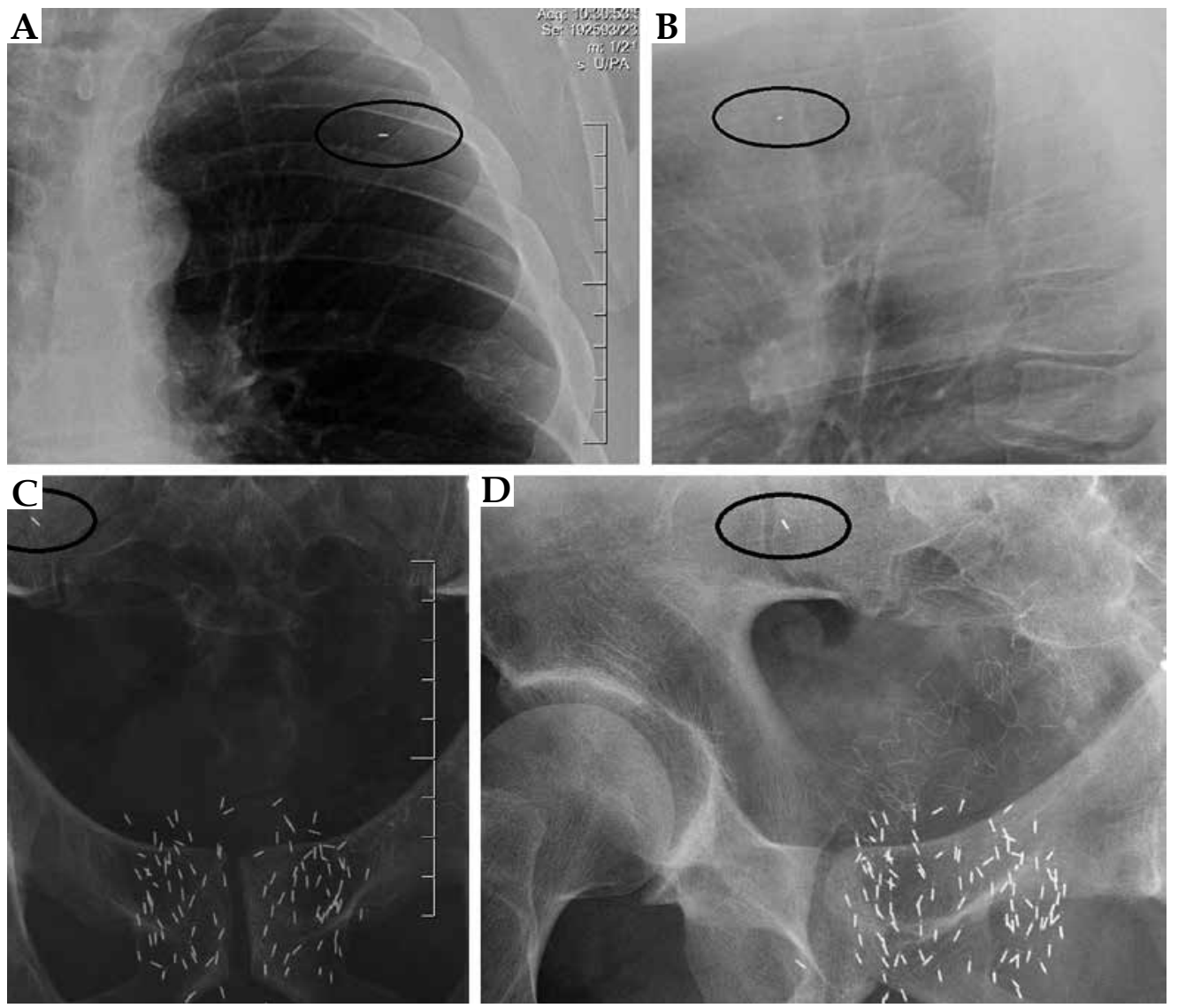

Fig. 1. Examples of seed loss as identified (circled) in the images one month post-implant: A) and B) to lung of one patient, and, C) and D) to pelvis of another patient. Shown on the left side of the figure (A and C) are the anterior-posterior images and the right side (B and $\mathrm{D})$ the lateral images

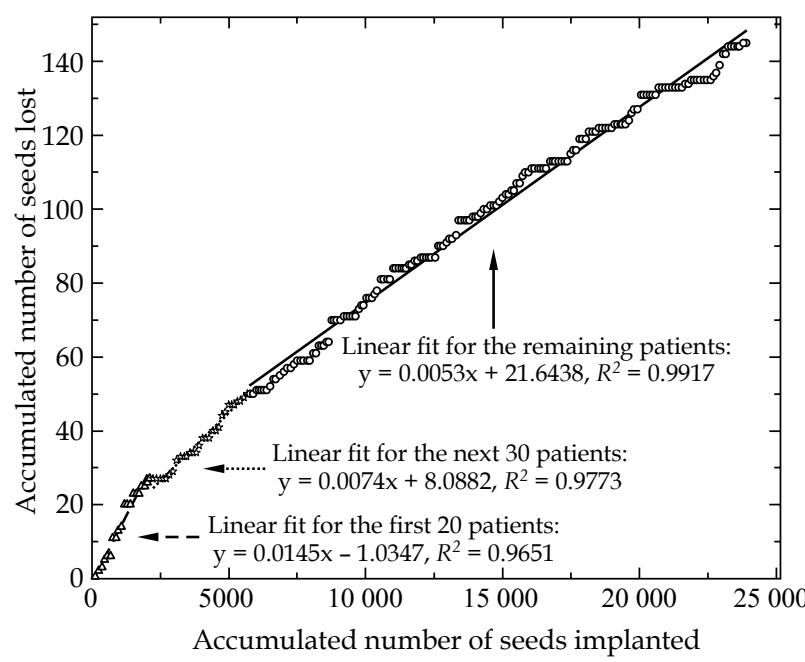

$\Delta$ First 20 patients Next 30 patients oRemaining patients

Fig. 2. The number of seeds lost against the number of seeds implanted with time. The slope of the linear fit was $0.0145,0.0074$, and 0.0053 for the first 20 , the next 30 , and the remaining patients, respectively, showing a trend toward the decrease the bias that may result from disparity in prostate lengths between pre- and post-implant images while calculating seed displacements. The mean prostate length $( \pm \mathrm{SD})$ was $39.7 \pm 5.1 \mathrm{~mm}$ (range: $26.0-56.1 \mathrm{~mm}$ ), and $39.3 \pm 4.7 \mathrm{~mm}$ (range: $27-57 \mathrm{~mm}$ ), pre- and post- implant, respectively $(p=0.4968)$. Most $(78.4 \%)$ of the prostate length differences between one month post-implant and pre-implant are within $3 \mathrm{~mm}$, while $19.8 \%$ of them are greater than $3 \mathrm{~mm}$ but within $6 \mathrm{~mm}$. There were only two $(1.8 \%)$ implants with a prostate length difference beyond $6 \mathrm{~mm}(6.5$ and $6.6 \mathrm{~mm}$, respectively). The mean prostate length difference $( \pm \mathrm{SD})$ was $-0.3 \pm 2.5 \mathrm{~mm}$.

The change in number of seeds inferior to the prostate from pre- to post-implant was studied to quantify the seed displacement. The mean number $( \pm \mathrm{SD})$ of the inferior seeds per patient was $9.2 \pm 2.2$ (range: 4 -14) in the pre-plan, compared to $8.2 \pm 4.5$ (range: 0 -30) one month post procedure.

The difference in the number of seeds inferior to prostate between pre-implant planning and post-implant dosimetry was $\leq 3$ seeds in $54.6 \%$ of patients, $\leq 6$ seeds in $86.6 \%$ of patients, and $\leq 7$ seeds in $93.1 \%$ of patients. There were two extreme cases; one patient had 11 less inferior seeds, while the other had 17 more inferior seeds in the post plans when compared to the pre-plans. The mean difference $( \pm S D)$ in the number of inferior seeds between 


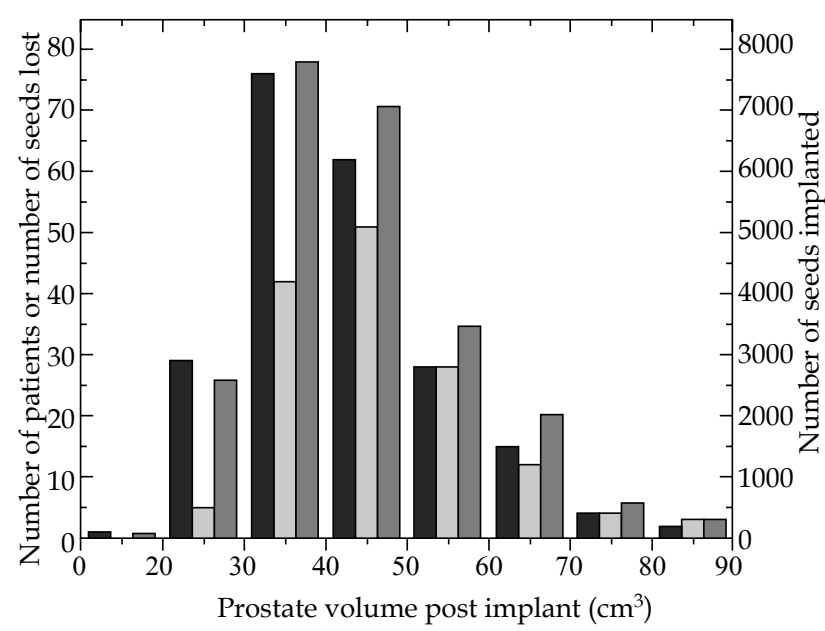

- Number of patients (scale on left vertical axis)

$\square$ Number of seeds lost (scale on left vertical axis)

$\square$ Number of seeds implanted (scale on right vertical axis)

Fig. 3. The number of patients, number of seeds lost, and number of seeds implanted versus the prostate volume post implant. The number of patients and seeds lost are shown in the left scale, while the number of seeds implanted is shown in the right scale. The horizontal axis is the prostate volume post implant with a bin size of $10 \mathrm{~cm}^{3}$

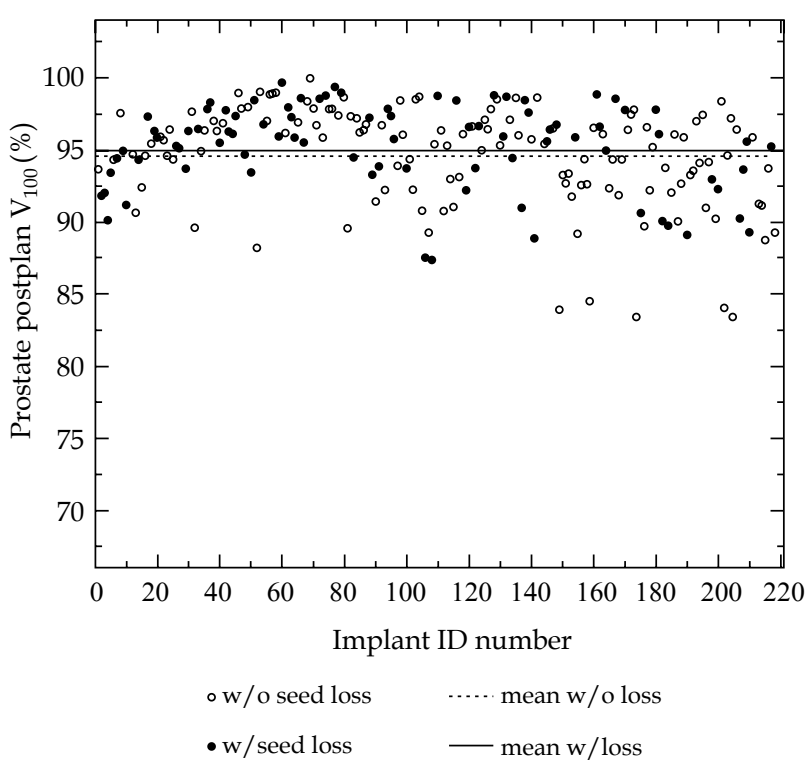

Fig. 5. Comparison of post plan prostate $V_{100}$ between implants without seed loss and those with seed loss. Also shown in the figure were the mean values for the two groups

pre-implantation plan and post-implant dosimetry was $-1 \pm 4.5$ seeds.

All seeds inferior to prostate apex were planned within $5 \mathrm{~mm}$ of prostate. At the implant procedure, $98.4 \%$ of these seeds were observed within $5 \mathrm{~mm}$ of the prostate apex, $99.8 \%$ of them were within $10 \mathrm{~mm}$, and $100 \%$ were within $15 \mathrm{~mm}$. The percentage of the seeds inferior to the

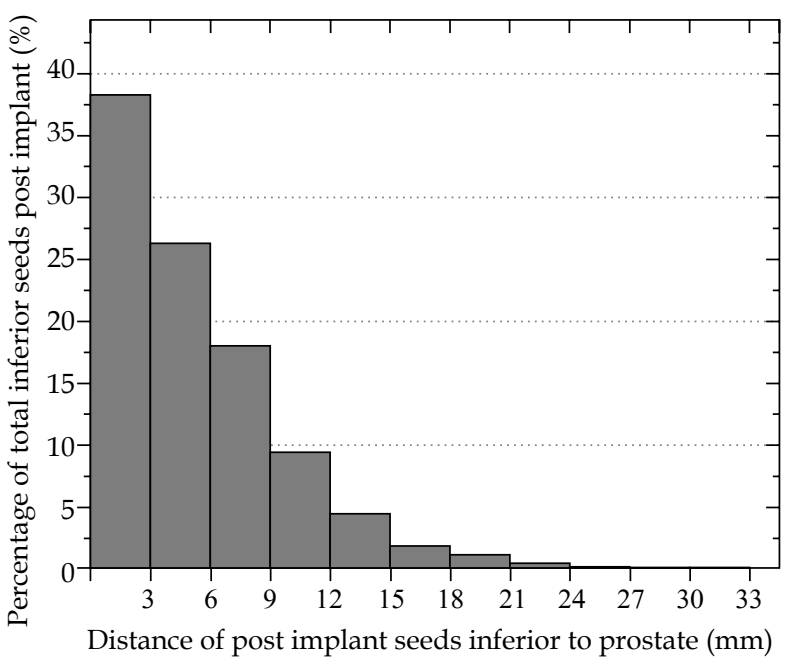

Fig. 4. The distribution of the distance of seeds inferior to the prostate as identified in the $\mathrm{CT} / \mathrm{MR}$ images one month post-implant

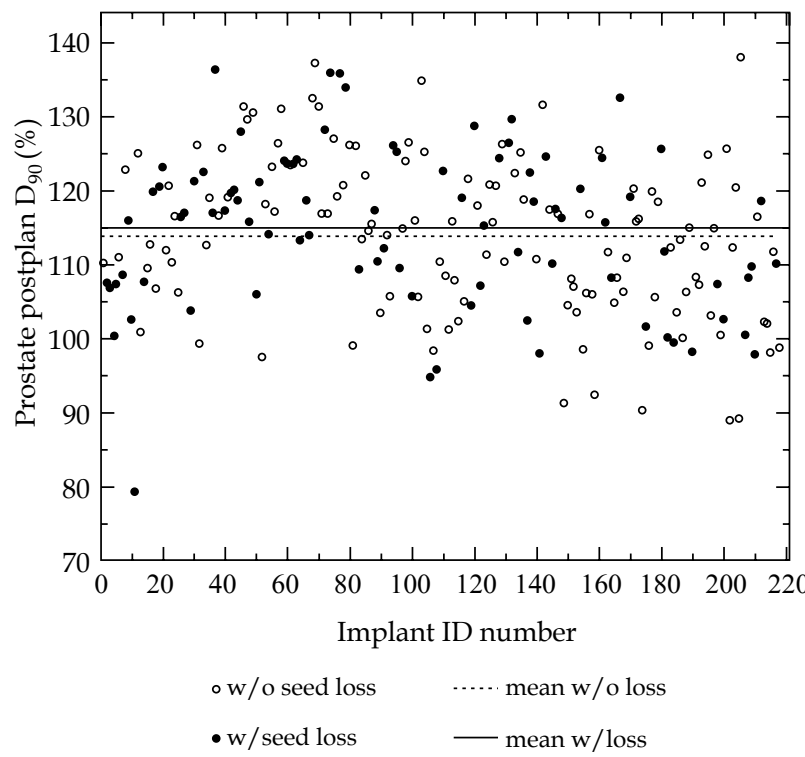

Fig. 6. Comparison of post plan prostate $\mathrm{D}_{90}$ between implants without seed loss and those with seed loss. Also shown in the figure were the mean values for the two groups

prostate as identified in the CT/MR images one month post-implant is shown as a function of distance from the prostate apex in Fig. 4. $96.3 \%$ of all inferior seeds were within $15 \mathrm{~mm}$ from the prostate apex. The remaining $3.7 \%$ of the inferior seeds were located more than $15 \mathrm{~mm}$ beyond the prostate, two seeds of which (each from a different patient) were $30 \mathrm{~mm}$ below the prostate. 
The one ( $0.056 \%$ of all inferior seeds) far most seed (found in another patient) was $33 \mathrm{~mm}$ inferior to the prostate apex.

\section{Dosimetric impact of seed loss and seed displacement}

\section{Seed loss}

For the whole population studied, the post-plan mean values $\left( \pm \mathrm{SD}\right.$ ) for prostate $\mathrm{V}_{100}$ and $\mathrm{D}_{90}$ were $94.7 \% \pm 3.8 \%$ and $114.2 \% \pm 10.6 \%$, respectively. To find out how seed loss affected post-implant prostate dosimetry, implants were divided into two groups, one without any seed loss and another with at least one seed lost. $V_{100}$ mean value for the group without seed loss $(n=130)$ was $94.6 \%$ with a standard deviation of $3.5 \%$ (range: from $83.4 \%$ to $99.9 \%$ ), while the mean value for the group with seed loss $(n=87)$ was $95.0 \%$ with a standard deviation of $4.2 \%$ (range: from $67.1 \%$ to $99.7 \%$ ). The difference in post-plan prostate $\mathrm{V}_{100}$ $(p=0.49)$ was not significant (Fig. 5).

The mean post-plan $\mathrm{D}_{90}( \pm \mathrm{SD})$ for the group without seed loss $(n=130)$ was $113.9 \% \pm 10.6 \%$ (range: $89.0-138.0 \%)$, compared to $114.8 \% \pm 10.7 \%$ (range: $79.4-136.4 \%$ ) in the group experiencing seed loss $(n=87)(p=0.52)$ (Fig. 6).

The rectal wall $\mathrm{RV}_{100}$ was 0.98 and $0.95 \mathrm{~cm}^{3}$ with a standard deviation 0.68 and $0.61 \mathrm{~cm}^{3}$ for the groups without and with seed loss, respectively $(p=0.80)$.

The post-implant prostate $\mathrm{V}_{100}, \mathrm{D}_{90}$, and rectal wall $\mathrm{RV}_{100}$ for the patient who lost 6 seeds (the maximum number of seeds lost per patient) out of 109 seeds implanted were $98.9 \%, 133.9 \%$, and $0.79 \mathrm{~cm}^{3}$, respectively. These dosimetry parameters for another patient who had 5 lost seeds out of 96 seeds inserted were $94.4 \%, 108.6 \%$, and $0.61 \mathrm{~cm}^{3}$, respectively. Both cases are considered acceptable.

\section{Seed displacement}

The mean percentage of changes in the number of seeds inferior to prostate (the change in the number of seeds inferior to prostate divided by the total seeds implanted) was $-1.1 \%$ with a standard deviation of $4.1 \%$, ranging from $-11.7 \%$ to $12.7 \%$. All extreme cases (with change beyond two times the standard deviation, or $8.2 \%$ ) were identified, and their inferior seeds and prostate $V_{100}$ and $\mathrm{D}_{90}$ post-implant along with their changes from the preplan were summarized in Table 2. The mean value of the post-plan prostate $V_{100}$ for these 12 cases was $94.7 \%$ with a standard deviation of $2.7 \%$ (a $5.0 \% \pm 2.3 \%$ decrease from the pre-plan), and was identical to the mean value for the whole population other than these 12 cases, i.e. $94.7 \% \pm 3.8 \%$ (a $5.2 \% \pm 3.8 \%$ decrease from the pre-plan). The mean post-plan $\mathrm{D}_{90}$ for these 12 cases was $114.7 \%$ with a standard deviation of $8.6 \%$ (a decrease of $9.3 \%$ $\pm 7.6 \%$ from the pre-plan) and was similar to the mean $\mathrm{D}_{90}$ for the whole population other than these 12 cases, i.e. $114.2 \% \pm 10.7 \%$ (a decrease of $10.6 \% \pm 10.0 \%$ ).

The mean rectal wall $\mathrm{RV}_{100}$ post-implant was 0.97 $\pm 0.58 \mathrm{~cm}^{3}$ for the 12 cases, similar to $0.97 \pm 0.66 \mathrm{~cm}^{3}$ for all other patients.

The 12 cases listed in Table 2 experiencing significant seed displacement were all acceptable and there were no differences from other cases studied, in terms of postplan prostate $V_{100}$ and $D_{90}$, and rectal wall $\mathrm{RV}_{100}$.

\section{Discussion and conclusions}

The seed loss and seed displacement of 217 consecutive patients treated using LDR brachytherapy were examined. Overall, $40.1 \%$ of patients experienced seed loss to lung, pelvis and abdomen, urine, and/or unknown destination, while seed migration to lung occurred in $15.5 \%$ of the patients. The mean number of seeds lost per patient was 0.67 with a standard deviation of 1.06 . The dosimetric impact of seed loss was insignificant when compared with implants without any seed loss.

The seed displacement in post-implant scans was minimal in terms of the displaced distance from prostate as well as the number of displaced seeds compared to pre-plan. The change in the number of seeds inferior to

Table 2. Post-plan dosimetry parameters for implants with significant seed displacement. Numbers in the brackets were the changes from pre-plan

\begin{tabular}{rccccc} 
Implant \# & \# Of inferior seeds & $\%$ Change of inferior seeds & Prostate $\mathrm{V}_{100}(\%)$ & Prostate $\mathrm{D}_{90}(\%)$ & Rectal wall RV $_{100}(\mathrm{cc})$ \\
\hline 16 & $3(-7)$ & -8.3 & $94.6(-5.4)$ & $112.8(-8.8)$ & $0.76(0.29)$ \\
\hline 22 & $24(+16)$ & 12.7 & $95.7(-4.3)$ & $120.7(-16.4)$ & $0.62(0.57)$ \\
\hline 27 & $19(+10)$ & 8.5 & $95.1(-4.9)$ & $116.9(-7.7)$ & $0.79(0.57)$ \\
\hline 72 & $16(+8)$ & 8.5 & $95.9(-4.1)$ & $116.9(-7.8)$ & $0.09(+0.08)$ \\
\hline 123 & $1(-7)$ & -8.5 & $95.0(-5.0)$ & $111.3(-11.6)$ & $0.43(+0.23)$ \\
\hline 125 & $0(-10)$ & -11.2 & $96.4(-3.6)$ & $115.7(-7.7)$ & $1.00(+0.95)$ \\
\hline 130 & $2(-9)$ & -8.5 & $96.0(-4.0)$ & $126.4(-2.4)$ & $1.98(+1.70)$ \\
\hline 140 & $1(-11)$ & -11.7 & $88.9(-8.8)$ & $98.0(-14.0)$ & $1.70(+1.44)$ \\
\hline 146 & $17(+11)$ & 9.4 & $96.5(-3.4)$ & $116.9(-4.2)$ & $1.00(+0.61)$ \\
\hline 196 & $30(+17)$ & 12.1 & $94.1(-5.6)$ & $114.9(-7.1)$ & $1.81(+0.86)$ \\
\hline 198 & $25(+14)$ & 12.6 & $90.2(-9.8)$ & $100.5(-27.0)$ & $0.55(+0.19)$ \\
\hline 200 & $4(-9)$ & -9.1 & $98.3(-1.6)$ & $125.7(-1.5)$ & $0.86(+0.23)$
\end{tabular}

$V_{100}$ - the percentage of the prostate volume receiving $100 \%$ of the prescribed dose or more, $D_{90}$ - the percentage of the prescribed dose received by $90 \%$ volume of the prostate, $R V_{100}$ - the absolute volume of the rectal wall to receive $100 \%$ of the prescription dose or more 
the prostate was within 7 seeds for $93 \%$ of patients, when compared to pre-plan. Eighty three percent of seeds inferior to prostate post-implant were within $9 \mathrm{~mm}$ from the prostate apex. There was no correlation between seed displacement and post-plan dosimetry in terms of prostate $\mathrm{V}_{100}$ and $\mathrm{D}_{90}$, and rectal wall $\mathrm{RV}_{100}$.

Despite our meticulous imaging with both MRI and CT scans for the dosimetry studies, in $20 \%$ of the patients, at least one seed went to unknown destination. This phenomenon is a significant contributor to the overall percentage of patients $(40.1 \%)$ who experienced seed loss. While a small uncertainty in seed counting may explain this difference in some of the patients, we believe that in many of these patients the seeds lost were excreted in the urine but not documented, which may not be always accounted to seeds lost in other study [4]. Seed migration to other organs not imaged is possible and is documented in rare case reports. The relative high number of seeds implanted due to the low seed activity may also increase the probability of seed loss [19]. Our overall seed loss percentage of patients is comparable to those reported in the literature [4,20-22].

In general, the number of seeds lost per patient increased with the prostate volume. This is understandable since the number of seeds implanted increased with prostate volume. The mean seed loss rate was $0.6 \%$ and is similar to that reported in the literature $[4,9,23]$.

Our study showed that neither seed loss nor seed displacement affects post implant prostate dosimetry. These results are consistent with the findings of Gao et al. [5] and Beaulieu et al. [19]. The possible reasons are the relative large number of seeds implanted, the optimal individual seed activity, the negligible mean number of seeds lost, and the insignificant seed displacement in terms of the displaced distance from prostate as well as the number of displaced seeds.

Pre-implant planning was performed utilizing US images, while post implant dosimetry used CT and MRI scans. This is actually the standard practice in most centers employing the pre-plan technique. Comparing images obtained by different modalities can be challenging, but as both US and MRI can identify the prostate boundaries with high accuracy, this comparison is suitable. Todor et al. described the post-implant intra-operative dosimetry using US images to identify the prostate, and cone beam CT to identify the seeds [24]. While that technique is promising, further data is needed before replacing the standard CT/MRI imaging with the US dosimetry.

While the dosimetry of the whole prostate is not affected by seed loss or migration, the dosimetry of specific sectors of the prostate may be affected. Several groups have previously shown that in I-125 seed brachytherapy, anterior prostate base receives a lower dose and apex receives a higher dose compared with pre-implant planned dose coverage [25-27]. Moreover, we have recently shown that the dose coverage of the clinical target volume of the prostate, defined as the prostate plus a $3 \mathrm{~mm}$ margin around it, is worse in patients with prostatic edema [28]. Clearly, seed loss in patients with prostatic edema would further worsen the dosimetry.
Regular chest X-ray imaging following LDR seed implantation is not a routine practice in most centers. The main reason that this is not done is the fact that there is not much to do once seed migration to lung is identified. Recent report about a non-smoker patient, who was diagnosed with small cell lung cancer 10 years after LDR prostate brachytherapy, with a brachytherapy seed detected in the surgical specimen, raises concern that seed migration to lung has potential consequence [29]. In this study, we showed that seed loss and migration rates were comparable to the ones reported in the literature and that it has no impact on the overall quality of the implant. Even though in this study, the implant quality was not affected by current rate of seed loss and displacements, we are now using all stranded seeds in order to avoid seed migration to critical organs.

\section{Acknowledgements}

This work was presented in part at the International Conference on Medical Physics Conference, Brighton, UK, September 1-4, 2013.

\section{Disclosure}

Authors report no conflict of interest.

\section{References}

1. Grimm PD, Blasko JC, Sylvester JE et al. 10-year biochemical (prostate-specific antigen) control of prostate cancer with ${ }^{125} \mathrm{I}$ brachytherapy. Int J Radiat Oncol Biol Phys 2001; 51: 31-40.

2. Crook J, Borg J, Evans A et al. 10-year experience with I-125 prostate brachytherapy at the Princess Margaret Hospital: results for 1,100 patients. Int J Radiat Oncol Biol Phys 2011; 80: 1323-1329.

3. Aronowitz JN, Rivard MJ. The phylogeny of permanent prostate brachytherapy. J Contemp Brachytherapy 2013; 5: 89-92.

4. Taussky D, Moumdjian C, Larouche R et al. Seed migration in prostate brachytherapy depends on experience and technique. Brachytherapy 2012; 11: 452-456.

5. Gao M, Wang J Z, Nag S et al. Effects of seed migration on post-implant dosimetry of prostate brachytherapy. Med Phys 2007; 34: 471-480.

6. Knaup C, Mavroidis P, Esquivel C et al. Investigating the dosimetric and tumor control consequences of prostate seed loss and migration. Med Phys 2012; 39: 3291-3298.

7. Pinkawa M, Gagel B, Asadpour B et al. Seed displacements after permanent brachytherapy for prostate cancer in dependence on the prostate level. Strahlenther Onkol 2008; 184: 520-525.

8. Fuller DB, Koziol JA, Feng AC. Prostate brachytherapy seed migration and dosimetry: analysis of stranded sources and other potential predictive factors. Brachytherapy 2004; 3: 10-19.

9. Saibishkumar EP, Borg J, Yeung I et al. Sequential comparison of seed loss and prostate dosimetry of stranded seeds with loose seeds in $125 \mathrm{I}$ permanent implant for low-risk prostate cancer. Int J Radiat Oncol Biol Phys 2009; 73: 61-68.

10. Kaplan ID, Meskell PM, Lieberfarb M et al. A comparison of the precision of seeds deposited as loose seeds versus suture embedded seeds: A randomized trial. Brachytherapy 2004; 3: 7-9.

11. Yu Y, Anderson L, Li Z et al. Permanent prostate seed implant brachytherapy: Report of the American Association of Physicists in Medicine Task Group No. 64. Med Phys 1999; 26: 2054-2076. 
12. Ishiyama $H$, Satoh $T$, Sekiguchi A. Comparison of three different techniques of low-dose-rate seed implantation for prostate cancer. J Contemp Brachytherapy 2015; 7: 3-9.

13. Taschereau R, Roy J, Pouliot J. Monte Carlo simulations of prostate implants to improve dosimetry and compare planning methods. Med Phys 1999; 26: 1952-1959.

14. Langley SE, Laing RW. 4D Brachytherapy, a novel real-time prostate brachytherapy technique using stranded and loose seeds. BJU Int 2012; 109 (Suppl 1): 1-6.

15. Rivard MJ, Butler WM, Devlin PM et al. American Brachytherapy Society recommends no change for prostate permanent implant dose prescriptions using iodine- 125 or palladium-103. Brachytherapy 2007; 6: 34-37.

16. Davis BJ, Horwitz EM, Lee WR et al. American Brachytherapy Society consensus guidelines for transrectal ultrasound-guided permanent prostate brachytherapy. Brachytherapy 2012; 11: 6-19.

17. Nath R, Bice WS, Butler WM et al. AAPM recommendations on dose prescription and reporting methods for permanent interstitial brachytherapy for prostate cancer: Report of Task Group 137. Med Phys 2009; 36: 5310-5322.

18. Morris WJ, Keyes M, Palma D et al. Evaluation of dosimetric parameters and disease response after ${ }^{125}$ Iodine transperineal brachytherapy for low- and intermediate-risk prostate cancer. Int J Radiat Oncol Biol Phys 2009; 73: 1432-1438.

19. Beaulieu L, Archambault L, Aubin S et al. The robustness of dose distributions to displacement and migration of ${ }^{125} \mathrm{I}$ permanent seed implants over a wide range of seed number, activity, and designs. Int J Radiat Oncol Biol Phys 2004; 58: 1298-1308.

20. Ankem MK, DeCarvalho VS, Harangozo AM et al. Implications of radioactive seed migration to the lungs after prostate brachytherapy. Urology 2002; 59: 555-559.

21. Eshleman JS, Davis BJ, Pisansky TM et al. Radioactive seed migration to the chest after transperineal interstitial prostate brachytherapy: extraprostatic seed placement correlates with migration. Int J Radiat Oncol Biol Phys 2004; 59: 419-425.

22. Nedea E, Wallner K, Reed D et al. Extraprostatic seed placement and its effect on seed loss. Cancer J 2005; 11: 147-151.

23. Sugawara A, Nakashima J, Kunieda E et al. Incidence of seed migration to the chest, abdomen, and pelvis after transperineal interstitial prostate brachytherapy with loose ${ }^{125}$ I seeds. Radiat Oncol 2011; 6: 130.

24. Todor DA, Zaider M, Cohen GN et al. Intraoperative dynamic dosimetry for prostate implants. Phys Med Biol 2003; 48: 1153-1171.

25. Nasser NJ, Wang Y, Borg J et al. Sector analysis of dosimetry of prostate cancer patients treated with low-dose-rate brachytherapy. Brachytherapy 2014; 13: 369-374.

26. Salembier C, Rijnders A, Henry A et al. Prospective multi-center dosimetry study of low-dose Iodine-125 prostate brachytherapy performed after transurethral resection. J Contemp Brachytherapy 2013; 2: 63-69.

27. Merrick GS, Butler WM, Grimm P et al. Permanent prostate brachytherapy extracapsular radiation dose distributions: analysis of a multi-institutional database. J Contemp Brachytherapy 2013; 3: 117-121.

28. Nasser NJ, Sappiatzer J, Wang Y et al. Dosimetric evaluation of clinical target volume in the postimplant analysis of lowdose-rate brachytherapy for prostate cancer. Brachytherapy 2015; 14: 189-196.

29. Chen WC, Katcher J, Nunez C et al. Radioactive seed migration after transperineal interstitial prostate brachytherapy and associated development of small-cell lung cancer. Brachytherapy 2012; 11: 354-358. 